\title{
Primeiro registro de Psyllaephagus bliteus parasitando Glycaspis brimblecombei em Chapadão do Sul, MS
}

\author{
Ricardo de Lima Vieira', Luis Gustavo Amorim Pessoa ${ }^{1}$, Valmir Antônio Costa ${ }^{2}$, Elisângela \\ de Souza Loureiro ${ }^{1}$, Nerison Luís Poersch ${ }^{1}$
}

\footnotetext{
${ }^{1}$ Universidade Federal de Mato Grosso do Sul, Campus de Chapadão do Sul, Chapadão do Sul, Mato Grosso do Sul, Brasil. E-mail: rflorestal@outlook.com, luis.pessoa@ufms.br, elisangela.loureiro@ufms.brnerison.poersch@ufms.br.

${ }^{2}$ Instituto Biológico, Campinas, São Paulo, Brasil. E-mail: valmir@biologico.sp.gov.br
}

Recebido: 09/03/2018; Aceito: 03/06/2018.

\section{RESUMO}

Psyllaephagus bliteus Riek, 1962 (Hymenoptera: Encyrtidae) é uma vespa de origem australiana que parasita ninfas de Glycaspis brimblecombei Moore, 1964 (Hemiptera: Psyllidae). No Brasil, a presença de ambos foi registrada pela primeira vez no Estado de São Paulo em 2003 e, posteriormente, sua ocorrência foi registrada nos estados do Paraná, Goiás, Minas Gerais, Santa Catarina, Rio Grande do Sul e Mato Grosso. No manejo dessa praga, $P$. bliteus é uma das formas mais promissoras de controle biológico clássico, uma vez que $G$. brimblecombei tem causando danos significativos na produção florestal. A ocorrência destes insetos na região nordeste de Mato Grosso do Sul foi constatada no Município de Chapadão do Sul, em povoamento de Eucalyptus camaldulensis, confirmando o potencial dispersivo e adaptativo de ambos.

Palavras-chave: psilídeo-de-concha; proteção florestal; entomologia florestal.

First record of Psyllaephagus bliteus parasitizing Glycaspis brimblecombei in Chapadão do Sul, MS, Brazil

\begin{abstract}
Psyllaephagus bliteus Riek, 1962 (Hymenoptera: Encyrtidae) is an Australian-origin wasp parasitizing nymph of Glycaspis brimblecombei Moore, 1964 (Hemiptera: Psyllidae). In Brazil, the presence of both was recorded for the first time in the State of São Paulo in 2003, and later its occurrence was recorded in the states of Paraná, Goiás, Minas Gerais, Santa Catarina, Rio Grande do Sul and Mato Grosso. In the management of this pest, $P$. bliteus is one of the most promising forms of classical biological control since G. brimblecombei has caused significant damage to forest production. The occurrence of these insects in the northeast region of Mato Grosso do Sul was verified in the Municipality of Chapadão do Sul, in a settlement of Eucalyptus camaldulensis, confirming the dispersive and adaptive potential of both.
\end{abstract}

Keywords: shell-shaped psyllid; forest protection; forestry entomology. 
O Estado de Mato Grosso do Sul apresenta cadeia produtiva de base florestal formada pela produção, distribuição e consumo de bens e serviços produzidos principalmente pelos segmentos de produção de celulose e papel, serrados, siderurgia a carvão vegetal e produção independente de madeira in natura. O Estado conta com aproximadamente 2,8 milhões de hectares de área plantada de eucalipto (IBGE, 2016).

Com a expansão da produção florestal em Mato Grosso do Sul, especificamente na silvicultura de eucalipto, têm sido relatados problemas com pragas florestais, principalmente com o psilídeo-de-concha Glycaspis brimblecombei Moore, 1964 (Hemiptera: Psyllidae) (Wilcken et al., 2003; Vieira et al., 2018). Trata-se de um inseto sugador exótico, nativo da Austrália, sendo registrada sua primeira ocorrência no Brasil em 2003, no Estado de São Paulo e rapidamente foi disperso para outros estados como Paraná, Goiás, Minas Gerais (Santana et al., 2003), Santa Catarina (Lutinski et al., 2006), Rio Grande do Sul (Oliveira et al., 2006) e Mato Grosso (Silva et al., 2013).

Glycaspis brimblecombei se caracteriza principalmente por atacar plantios jovens de eucalipto, causando danos como: deformações e queda prematura de folhas, favorecimento do aparecimento de fumagina, seca de brotos terminais e redução da área fotossintética devido ao elevado número de conchas, que são formadas pela excreção do inseto, as quais abrigam as ninfas até o estádio adulto (Santana et al., 2003; Wilcken et al., 2003).

A presença de seu inimigo natural, o endoparasitoide solitário Psyllaephagus bliteus Riek, 1962 (Hymenoptera: Encyrtidae), que é uma vespa exótica nativa da Austrália, está diretamente relacionada com os níveis populacionais dessa praga; seu ciclo biológico completo ocorre entre três e cinco semanas em qualquer época do ano, semelhante ao de seu hospedeiro (Sá \& Wilcken, 2004; Ferreira et al., 2009). O parasitismo pode ser percebido facilmente devido à presença de um orifício na região lateral da concha, realizado quando da emergência do adulto que perfura o abdome da ninfa parasitada até a superfície da concha (Wilcken et al., 2010).

Embora a presença de $P$. bliteus seja conhecida no Brasil desde 2003 (Berti Filho et al., 2003), a forma na qual o parasitoide e seu hospedeiro foram introduzidos no país ainda é incerta, pela sua rápida capacidade de dispersão no ecossistema florestal, favorecido pela facilidade adaptativa às condições climáticas no Brasil $\mathrm{e}$ pela grande extensão de povoamentos com eucalipto (Lutinski et al., 2006).

Na região nordeste do Estado de Mato Grosso do Sul foi observado o parasitismo de ninfas de $G$. brimblecombei, em folhas de Eucalyptus camaldulensis Dehnh, com nove meses de idade e altura média de dois metros, disposto num arranjo espacial de 3,0 × 2,0 m, em uma área de 0,7 hectares no Campus Experimental da Universidade Federal de Mato Grosso do Sul (UFMS), Campus de Chapadão do Sul (CPCS) (18 47'39', S, 52॰37’22'” W), sem tratamento fitossanitário desde a implantação em campo. As plantas são dispostas em quatro blocos contendo 20 plantas cada. Foram escolhidas aleatoriamente cinco plantas em cada bloco as quais foram marcadas com auxílio de barbante preso ao caule.

O solo da área de estudo foi classificado como Latossolo Vermelho distrófico e o clima da região é do tipo tropical úmido (Aw), com estação chuvosa no verão e seca no inverno, segundo Köppen. A temperatura média anual varia de $13^{\circ}$ a $28^{\circ} \mathrm{C}$ e a precipitação média anual é de $1.850 \mathrm{~mm}$.

Semanalmente eram coletadas, aleatoriamente, 20 folhas de cada planta, contendo ninfas do psilídeo (Figura 1), totalizando 400 folhas por amostragem, no período de setembro a outubro de 2014. As folhas eram acondicionadas em sacos plásticos transparentes e levadas até o Laboratório de Entomologia da UFMS / CPCS, sendo armazenadas em sacos de papel kraft, com um tubo de ensaio transparente preso a sua abertura, para coleta dos insetos. Posteriormente, em laboratório, era contabilizado o número de psilídeos-de-concha parasitados e não parasitados por folha, retirando-se os parasitados após a contagem. Também foram coletadas folhas contendo psilídeos para verificar a emergência do parasitoide.

Os parasitoides obtidos tiveram o gênero e a espécie determinado com auxílio dos trabalhos de Noyes et al. (1997) e de Riek (1962) e Berry (2007), sendo identificados como $P$. bliteus. Os insetos encontram-se depositados no Laboratório de Entomologia do CPCS e na Coleção de Insetos Entomófagos "Oscar Monte”, do Instituto Biológico de Campinas, SP.

A ocorrência do parasitoide já foi registrada nos Estados de São Paulo (Berti Filho et al., 2003), Santa Catarina (Garcia et al., 2011) e Mato Grosso (Silva et al., 2013). P. bliteus apresenta coloração verde metálico, pernas de cor de creme, asas hialianas e antenas geniculadas com 12 segmentos nas fêmeas e 10 segmentos nos machos, apresentando dimorfismo sexual (Riek, 1962). As fêmeas medem cerca de 1,88 a 2,36 $\mathrm{mm}$ de comprimento, com antenas de coloração marrom, diferentemente dos machos que medem cerca de 1,63 a $1,97 \mathrm{~mm}$ de comprimento e possuem antenas de coloração amarela.

Apesar das características morfológicas mencionadas acima, o parasitismo de $P$. bliteus pode ser facilmente observado, devido à presença de um orifício na concha, realizado quando da emergência do adulto (Figura 1). 

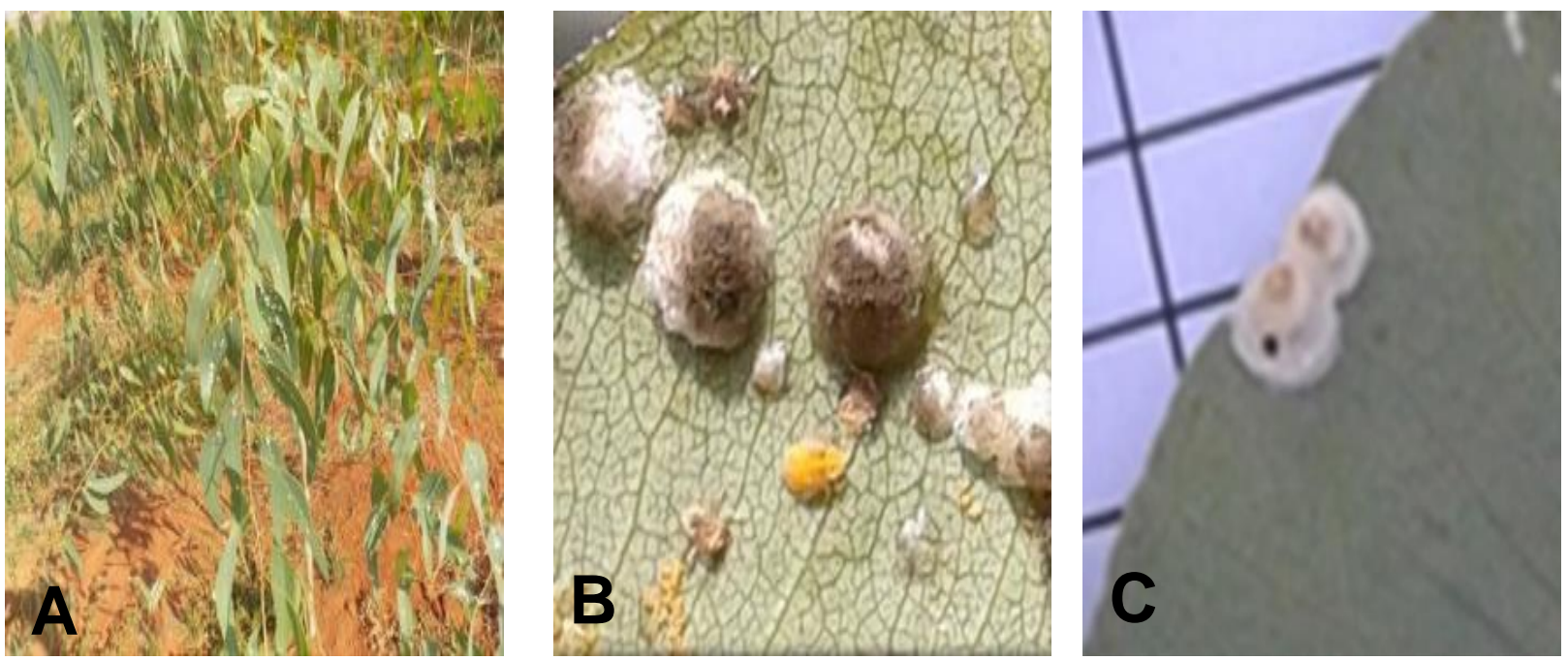

Figura 1. A- Eucalyptus camaldulensis infestadas com G. brimblecombei; B- Ninfas de G. brimblecombei; C- Detalhe do orifício de saída de $P$. bliteus após ocorrer o parasitismo.

Verificaram-se taxas de parasitismo variando de aproximadamente $60 \%$ no mês de setembro a $7 \%$ no mês de outubro, sendo que em ambos os períodos essa taxa chegou a $0 \%$ (Figura 2). Este fato pode estar relacionado com os fatores climáticos da região, principalmente a pluviosidade, obtida pela estação meteorológica da Fazenda Campo Bom (1843'39' S, $\left.52^{\circ} 36^{\prime} 05^{\prime \prime} \mathrm{W}\right)$, que atua como regulador populacional de G. brimblecombei, como verificado por Ferreira et al. (2009) e Silva et al. (2013).

Essa variação também foi relatada em estudos conduzidos nos EUA e México, em eucalipto, onde a taxa de parasitismo no campo variou de 7 a $92 \%$, respectivamente (Garrison, 2001). Estudos de flutuação populacional desses insetos no campo têm sido fundamental para a aplicação do controle biológico clássico, devido a variações na taxa de parasitismo, ocasionadas por gerações normais, com machos e fêmeas, e gerações partenogenéticas, com apenas machos, o que reduz a eficiência do parasitoide (Silva et al., 2013).

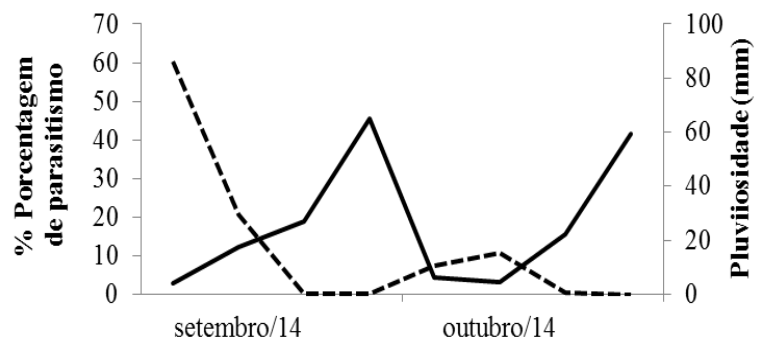

Período de avaliação

---. \% parasitismo — Pluviosidade
Figura 2. Parasitismo de Psyllaephagus bliteus em ninfas de Glycaspis brimblecombei no período de setembro a outubro de 2014, em Chapadão do Sul, MS.

O controle biológico clássico é, dentre as possíveis formas de controle do psilídeo-de-concha, a mais promissora, oferecendo menores custos e menor impacto ambiental devido à especificidade de seu inimigo natural (Garcia et al., 2011).

Apesar de a região nordeste de Mato Grosso do Sul não se caracterizar como a principal região produtora de eucalipto no Estado, os Municípios de Chapadão do Sul, Cassilândia, Costa Rica e Paraiso das Águas, geograficamente próximos e localizados na referida região, apresentam 44.000 ha cultivados com eucaliptos (IBGE, 2016). Assim, a ocorrência natural de P. bliteus o destaca como um agente promissor no controle de $G$. brimblecombei, devido às altas taxas de parasitismo verificadas em campo.

\section{Referências Bibliográficas}

Berti Filho, E., Costa, V. A., Zuparko, R. L., Lasalle, J.,2003. Ocorrência de Psyllaephagus bliteus Riek (Hymenoptera: Encyrtidae) no Brasil. Revista de Agricultura, 78, 304.

Berry, J. A., 2007. Key to New Zealand species of Psyllaephagus Ashmead (Hymenoptera: Encyrtidae) with descriptions of three new species and a new record of the psyllid hyperparasitoid Coccidoctonus psyllae Riek (Hymenoptera: Encyrtidae). Australian Journal of Entomology, 46, 99-105.

Ferreira, A. R., Blaziza, B. A. A., Anzolin, G. M., WincklerFirmino, C. D., 2009. Flutuação populacional do psílideo-deconcha Glycaspis brimblecombei Moore (Hemiptera: Psyllidae) em Eucalyptus spp. no município de Garça, SP. Revista Eletrônica de Engenharia Florestal, ano VIII, n. 14. 
Garcia, M. R. F., Savaris, M., Pereira, M. V. D., 2011. Primeiro registro do parasitoide Psyllaephagus bliteus Riek (Hymenoptera, Encyrtidae) no estado de Santa Catarina, Brasil. Biodiversidade Pampeana, 9, 61-63.

Garrison, W., 2001. New agricultural pest for Southern California: Spotted Gum Lerp Psyllid, Eucalyptolyma maideni. San Diego County Agricultural Commissioner's office.Disponível em:http://www.sandiegocounty.gov/reusable_components/ima gens/awm/Docs/ipd_spottedpsyllid.pdf. Acesso em: 07 de maio de 2018.

Lutinski, J. A., Lutinski, J. C., Garcia, F. R. M, 2006. Primeiro registro de Glycaspis brimblecombei Moore 1964, (Hemiptera: Psyllidae) em Eucalipto no Estado de Santa Catarina, Brasil. Ciência Rural, 36, 653655.

Noyes, J. S., Woolley, J. B., Zolnerowich, G. Encyrtidae., 1997. In: Gibson, G. A. P., Huber, J. T., Woolley, J. B. (Eds.) Annotated keys to the genera of Nearctic Chalcidoidea (Hymenoptera). NRC Research Press, 170-320.

Oliveira, L. O., Costa, E. C., Grellman, M., Cantarelli, E. B., Perrando, E. R., 2006. Ocorrência de Glycaspis brimblecombei Moore 1964, (Hemiptera: Psyllidae) em Eucalyptus spp. no Rio Grande do Sul, Brasil. Ciência Florestal, 16, 353-355.

Riek, E. F., 1962. The Australian species of Psyllaephagus (Hymenoptera: Encyrtidae), parasites of psyllids (Homoptera). Australian Journal of Zoology, 10, 684-757.

Sá, L. A. N., Wilcken, C. F., 2004. Nova praga exótica no ecossistema florestal. Embrapa Meio Ambiente, 3.
Santana, D. L. Q., Menezes Junior, A., Silva, H. D., Bellote, A. F. G., Favaro, R. M., 2003. O Psilídeo-de-concha (Glycaspis brimblecombei) em Eucalipto. Embrapa Florestas, 3.

Silva, A. L., Peres-Filho, O., Dorval, A., Castro, C. K. C., 2013. Dinâmica Populacional de Glycaspis brimblecombei e inimigos naturais em Eucalyptus spp., Cuiabá-MT. Floresta e Ambiente, 20, 80-90.

Vieira, R. L., Pessoa, L. G. A., Loureiro, E. S., Poersh, N. L., 2018. Ocorrência de Glycaspis brimblecombei sobre Eucalyptus em Chapadão do Sul, Mato Grosso do Sul. Revista de Agricultura Neotropical, 5, 94-96.

Wilcken, C. F., Coutro, E. B., Orlato, C., Ferreira Filho, P. J., Firmino, D. C., 2003. Ocorrência do Psilídeo-de-concha (Glycaspis brimblecombei) (Hemiptera: Psylladae) em florestas de eucalipto no Brasil. Instituto de Pesquisas e Estudos Florestais, 11.

Wilcken, C. F., Sá, N. A. L., Pogetto, D. A. F. H. M., Couto, B. E., Filho, F. J. P., Winckler-Firmino, C. D., 2010. Sistema de criação do psilídeo-de-concha Glycaspis brimblecombei (Hemiptera: Psyllidae) e de seu parasitoide Psyllaephagus bliteus (Hymenoptera: Encyrtidae) para programa de controle biológico em plantações de eucalipto. Documentos Técnicos IPEF, 2, 1-23.

IBGE, 2016. Instituto Brasileiro de Geografia e Estatística. Produção da Extração vegetal e da Silvicultura, 31, 1-54. Disponívelem:https://biblioteca.ibge.gov.br/visualizaçao/perio dicos/74/pevs_2016_v31.pdf. Acesso em: 06 de maio 2018. 